\title{
Strain Energy Harvesting Powered Wireless Sensor System Using Adaptive and Energy- Aware Interface for Enhanced Performance
}

\author{
Zheng Jun Chew, Member, IEEE, Tingwen Ruan, and Meiling Zhu, Member, IEEE
}

\begin{abstract}
This paper presents a wireless sensor system (WSS) powered by a strain energy harvester (SEH) through the introduction of an adaptive and energy-aware interface for enhanced performance under variable vibration conditions. The interface is realized by an adaptive power management module (PMM) for maximum power transfer under different loading conditions and an energy-aware interface (EAI) which manages the energy flow from the storage capacitor to the WSS for dealing with the mismatch between energy demanded and energy harvested. The focus is to realize high harvested power and high efficiency of the system under variable vibration conditions, and an aircraft wing structure is taken as a study scenario. The SEH powered WSS was tested under different peak-to-peak strain loadings from 300 to $600 \mu \varepsilon$ and vibrational frequencies from 2 to $10 \mathrm{~Hz}$ to verify the system performance on energy generation and distribution, system efficiency, and capability of powering a custom-developed WSS. Comparative studies of using different circuit configurations with and without the interface were also performed to verify the advantages of the introduced interface. Experimental results showed that under the applied loading of $600 \mu \varepsilon$ at $10 \mathrm{~Hz}$, the SEH generates $0.5 \mathrm{~mW}$ of power without the interface while having around $670 \%$ increase to $3.38 \mathrm{~mW}$ with the interface, which highlights the value of the interface. The implemented system has an overall efficiency of 70 to $80 \%$, a long active time of more than $1 \mathrm{~s}$, and duty cycle of up to $11.85 \%$ for vibration measurement under all the tested conditions.
\end{abstract}

Index Terms-adaptive power management, energy-aware interface, piezoelectric energy harvesting, strain energy harvester, wireless sensor node.

\section{INTRODUCTION}

$\mathrm{W}$ IRELESS SENSOR NETWORKS (WSNs) that are usually embedded within or installed on engineering structures [1] are demanding alternative energy sources to batteries to power up the system because battery-powered WSNs require more routine maintenances just for the batteries replacement than the structures themselves need, which is not sensible. Therefore, energy harvesting which converts sources from the ambient environments such as radio-frequency energy, solar

This work was supported in part by the Engineering and Physical Sciences Research Council, U.K., through the project En-ComE-Energy Harvesting Powered Wireless Monitoring Systems Based on Integrated Smart Composite Structures and Energy-Aware Architecture under Grant EP/K020331/1. All data are provided in full in the results section of this paper. energy, thermal energy, and vibration energy [2]-[4] into electrical energy to power WSNs is highly sought for as a more durable and cost effective long term solution.

Vibration energy has been recognized as one of the best sources to power WSNs because of its wide availability in engineering structures such as moving vehicle with vibration, for example, aircraft [5]. Piezoelectric energy harvesters (PEHs) are particularly attractive to vibration energy harvesting because of their simple structures, self-contained power generation capabilities and high energy densities [5]. PEHs using flexible piezoelectric materials such as polyvinylidene fluoride (PVDF) [6] and macro fiber composite (MFC) [7] are particularly suitable as a strain energy harvester (SEH) because they can be bonded onto host structures of different shapes [7], [8], where the strain experienced by the host structures due to impact or vibration can be directly transferred to and converted by the SEH into electrical energy at off-resonant. The power that can be harvested is proportional to the vibration frequency and has a quadratic relationship with the experienced strain level [9].

Although researches in the use of SEHs have been rather intensive recently, not many studies on integrated systems of SEHs powered WSNs that are potentially suitable for real industrial applications under variable vibration conditions have been reported so far. Most of the work focused on the characterization of the SEH itself [6], [7] or together with a simple interface [8], [10] without connecting to a wireless sensor system (WSS) for a whole system study. An integrated whole system study of the energy harvester, system interface, and WSS under an intended operational condition is essential for research on energy harvesting powered WSS since a high performance of one subsystem does not guarantee a successful operation of the whole system. This is because of: (1) limited energy generated by harvesters, (2) high power consumption of energy harvesting circuitries and end devices, and (3) limited adaptation to intended application situations (variable ambient vibration environments and variable connected electric loads) of the circuitries. This means that (1) there is a mismatch between the energy generated by the harvesters and the energy demanded by the WSNs to perform required tasks, and (2) power delivered to the end devices is extremely low because of the lack of adaptation functionality of the simple interfaces, leading to an extremely low efficient operation. A typical example is that when a peak strain loading of $305 \mu \varepsilon$ at 
$2.5 \mathrm{~Hz}$ was applied, a MFC connected to its optimal resistive load can generate more than $1 \mathrm{~mW}$ of power, but when it was connected to a storage capacitor together with a WSS via a rectifier, only around $0.28 \mathrm{~mW}$ of power was generated [9]. This is because the simple interface of the rectifier itself is without adaptation functionality to variable vibrations and variable loads, and thus not able to harvest the power generated by the MFC which is subject to variable vibrational conditions and variable connected loads effectively.

There are only a few whole system integration studies on SEH powered WSS, most notably by Giuliano et al. [9] and MicroStrain Inc. [11]. The presented systems have common features, which include (1) charging up a storage capacitor using the SEH via a rectifier which is non-adaptive and (2) an energy-aware interface (EAI) that keeps the WSS off so that energy from the SEH can be accumulated in the storage capacitor and only allows the WSS to draw current from the storage capacitor when the stored energy is sufficiently high. The reported EAIs have low current consumption of $1.19 \mu \mathrm{A}$ [9] or lower [11] but the SEH harvested less than $1 \mathrm{~mW}$ [9], [11] even when a peak strain loading of $300 \mu \varepsilon$ at $180 \mathrm{~Hz}$ was applied because of the non-adaptive interface used.

The sizes of storage capacitors used in these studies are also small at less than $2 \mathrm{mF}$, which can only keep the sensor on for a few hundreds of milliseconds at most [9], [11]. Such a short turn-on time is usually insufficient especially for real-world applications such as structural health monitoring which requires monitoring of low to medium frequency vibrations using an accelerometer. For example, general mechanical loading such as human walking or vehicle moving on a bridge structure is less than $10 \mathrm{~Hz}$ [12], [13] and can be as low as $0.44 \mathrm{~Hz}$ [13]. Also, to be able to detect unexpected incident caused by either intentional damage or accidental events such as wear and tear of the structure or natural disaster which can be signified by the anomalous vibration measured, the WSS needs to be turned on for as long as possible and also as frequently as possible to measure and transmit the measured dynamic signals. Therefore, there is a real need for an energy harvesting interface between energy harvesters and end devices, not only to perform well in general duties of rectification, regulation and energy storage but also equip with adaptation and energy-aware functionalities to harvest as much energy as possible for shortening the idle time and prolonging the active time of a WSS to meet the aforementioned demands for real applications.

This paper herein presents a SEH powered WSS through a whole system study approach and introduces an adaptive and energy-aware interface between the SEH and WSS for enhanced performance, including an operation time of more than 1 second for real-world applications, where an aircraft wing structure is taken as a study scenario. The interface combines an adaptive power management module (PMM), which is capable to harvest energy from the SEH at maximum power point (MPP) under variable vibration conditions and an energy-aware interface (EAI) which is capable to deal with the energy mismatch by managing the energy flow from the storage capacitor to the WSS by allowing the harvested energy from the harvester to be accumulated in the storage capacitor. Comparative studies of the WSS powered by the SEH using different circuit configurations were conducted to determine the performance of the implemented system and evaluate the significances of the adaptive and energy-aware interface in enhancing the whole system performances. The energy distribution, efficiency, and wireless sensor operation of the implemented system were analyzed to study the whole system performance. Different sizes of storage capacitors were also used in this study to determine their influence on the operation of the WSS. Therefore, this paper provides a roadmap for a realistic implementation of an energy harvesting powered WSN for real-world applications.

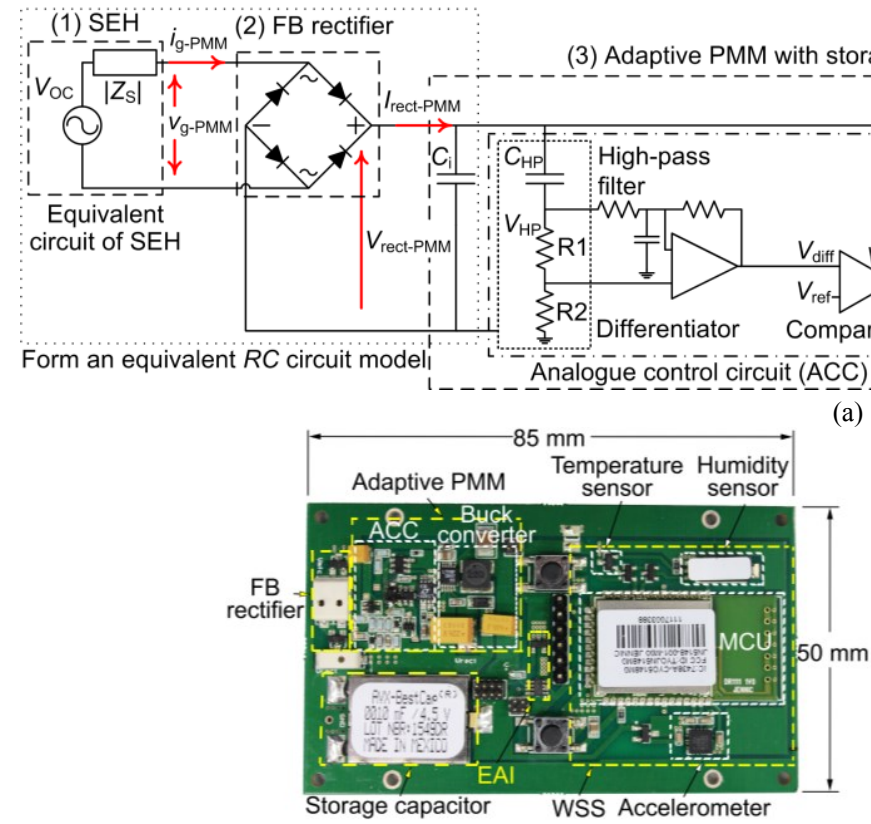

(b)
(4) EAI

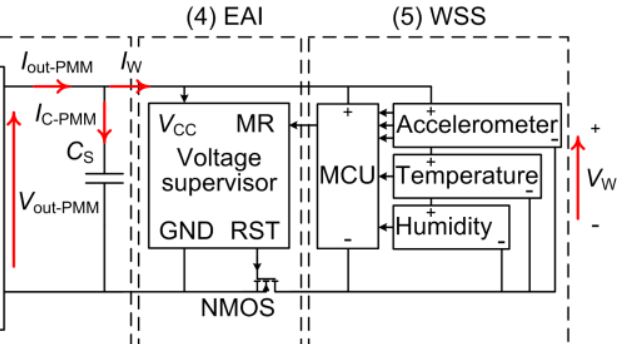

(a)

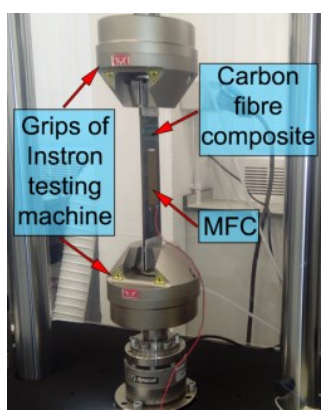

(c)

Fig. 1. (a) Circuit diagram of the SEH powered WSS, (b) the implemented system on a PCB, and (c) the manufactured SEH tested by Instron testing machine. 


\section{SEH POWERED WIRELESS SENSOR SYSTEM}

Fig. 1(a) shows the circuit diagram of a SEH powered WSS studied in this paper. The system consists of five main functional blocks namely, (1) a SEH for harvesting strain energy generated from aircraft wing vibrations, (2) a full wave diode bridge $(\mathrm{FB})$ rectifier for converting the $\mathrm{AC}$ voltage generated by the SEH into a DC voltage, (3) an adaptive PMM for MPP finding under variable vibration conditions and transferring the energy from the SEH to the storage capacitor $C_{\mathrm{S}}$ for energy accumulation using a buck converter, (4) an EAI for adaptive energy flow management between the storage capacitor and the WSS, and (5) a WSS for sensing tasks. The key functional blocks of the developed system are the analogue control circuit (ACC) of the adaptive PMM and the EAI which enable the SEH to power the WSS with high efficiency under variable vibration conditions. The SEH is specifically chosen for aircraft wing structural energy harvesting because of its lightweight, low profile, and ease of use without any additional mass added to the wing [9], [11]. The other four functional blocks in the system are designed for general purposes, where a prototype was fabricated with all the components populated on one side of a name card sized printed circuit board (PCB), as shown in Fig. 1(b).

\section{A. Strain Energy Harvester}

The aircraft wing experiences dynamic strain due to structural vibrations [7]. The MFC can be easily integrated in the inner side of the aircraft wing to convert the mechanical strain energy into electrical energy for powering WSNs because of its flexibility and thinness of $0.3 \mathrm{~mm}$. In this paper, the MFC M8528-P2 piezoelectric element (Smart Material GmbH, Dresden, Germany) [9], [14] is chosen as the SEH and is bonded to a carbon fiber composite, which is a modern aircraft material. The MFC has an active area of $85 \times 28 \mathrm{~mm}^{2}$ while the carbon fiber composite has a length, width, and thickness of $300 \mathrm{~mm}, 50 \mathrm{~mm}$, and $5 \mathrm{~mm}$, respectively. The manufactured carbon fiber composite material with the MFC bonded on its center region is shown in Fig. 1(c) with the Instron testing machine used for applying a strain loading onto the carbon fiber composite material with the bonded MFC.

\section{B. Adaptive Power Management Module}

As shown in Fig. 1(a), the adaptive PMM is composed of (1) an ACC and (2) a buck converter (LTC3388-3) to find the MPP for high energy transfer from the SEH under variable vibration conditions. The fundamental principle of the adaptive PMM is based on the well-known maximum power occurring at the magnitude of half-open-circuit voltage $\left(V_{\mathrm{OC}} / 2\right)$ of the PEH [15], [16] but the design and implementation of the ACC in the developed system is based on the literature [17].

The implemented method exploits the $R C$ response of the circuit to determine if the voltage across the smoothing capacitor is charged up to $V_{\mathrm{OC}} / 2$ using a specifically designed high-pass filter. The filter outputs a peak voltage that corresponds to the $V_{\mathrm{OC}} / 2$ of the $\mathrm{PEH}$, which is also the voltage when maximum power transfer occurs in that circuit configuration. Therefore, in the implemented adaptive PMM,

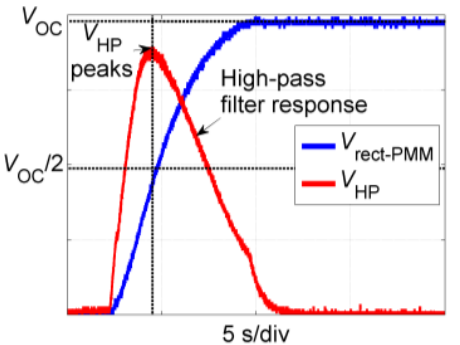

Fig. 2. Illustration of high-pass filter response of the ACC for MPP finding.

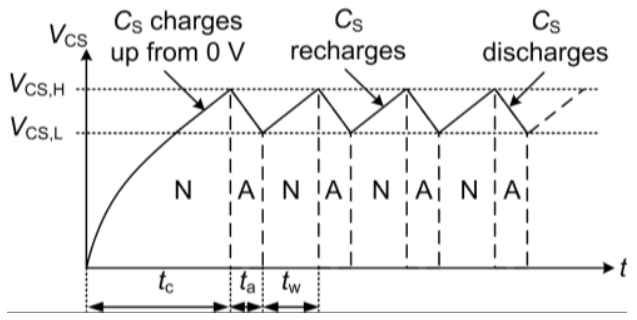

Fig. 3. Illustration of the voltage across the storage capacitor as controlled by the EAI.

the ACC simply finds the $V_{\mathrm{OC}} / 2$ of the $\mathrm{SEH}$ by tracking the rectified voltage $V_{\text {rect-PMM }}$ charging profile at $C_{\mathrm{i}}$ through the use of a high-pass filter and a differentiator, as shown in Fig. 1(a).

Initially, the buck converter is disabled and can be regarded as open-circuited so that $V_{\text {rect-PMM }}$ can increase to a magnitude which is equal to $V_{\mathrm{OC}}$ of the $\mathrm{SEH}$, assuming that the rectifier is lossless. A simple $R C$ circuit is formed by the modulus of the impedance of the $\mathrm{SEH}$ and $C_{\mathrm{i}}$ when the buck converter is disabled. The $R C$ high-pass filter formed by the capacitor $C_{\mathrm{HP}}$ and resistors $\mathrm{R} 1$ and $\mathrm{R} 2$ is used to track the voltage changes of $V_{\text {rect-PMm }}$ at $C_{\mathrm{i}}$. The high-pass filter is specifically designed to have a voltage output $V_{\text {HP }}$ reaching its peak when $V_{\text {rect-PMM }}$ reaches $V_{\mathrm{OC}} / 2$ as shown in Fig. 2. The output of the differentiator is zero when $V_{\mathrm{OC}} / 2$ is reached because the differentiation of maxima is zero. A comparator compares $V_{\text {diff }}$ with a reference signal $V_{\text {ref }}$ of slightly larger than zero since it is difficult for a comparator to compare a zero value with a reference which is zero as well where the output could be unpredictable. When $V_{\text {diff }}$ is smaller than $V_{\text {ref, }}$, the comparator sends out a signal pulse $V_{\text {comp }}$ to enable the buck converter for energy transfer from the $\mathrm{SEH}$ to the storage capacitor $C_{\mathrm{S}}$. Because of the energy transfer, $V_{\text {rect-PMM }}$ decreases and moves away from $V_{\mathrm{OC}} / 2$. Consequently, the values of $V_{\mathrm{HP}}$ and $V_{\text {diff }}$ change and the buck converter is disabled. $V_{\text {rect-PMM }}$ can then increase towards $V_{\mathrm{OC}} / 2$ for the next energy transfer process to start again and the cycles repeat as long as there is vibration applied onto the SEH.

\section{Energy-aware Interface}

As shown in Fig. 1(a), the EAI is a circuit (hardware) for energy flow management from the storage capacitor $C_{\mathrm{S}}$ to the WSS. The function of the EAI is to ensure that there is sufficient energy in $C_{\mathrm{S}}$ for the operation of the WSS before releasing it to the WSS. This means no energy is consumed by the WSS when there is insufficient energy, which allows the energy transferred from the SEH by the adaptive PMM to be accumulated in $C_{\mathrm{S}}$. As the energy stored in $C_{\mathrm{S}}$ can be related to the voltage $V_{\mathrm{CS}}$ of $C_{\mathrm{S}}$, the EAI is realized using a voltage supervisor (LTC2935-1) to monitor $V_{\mathrm{CS}}$ and toggle the NMOS 


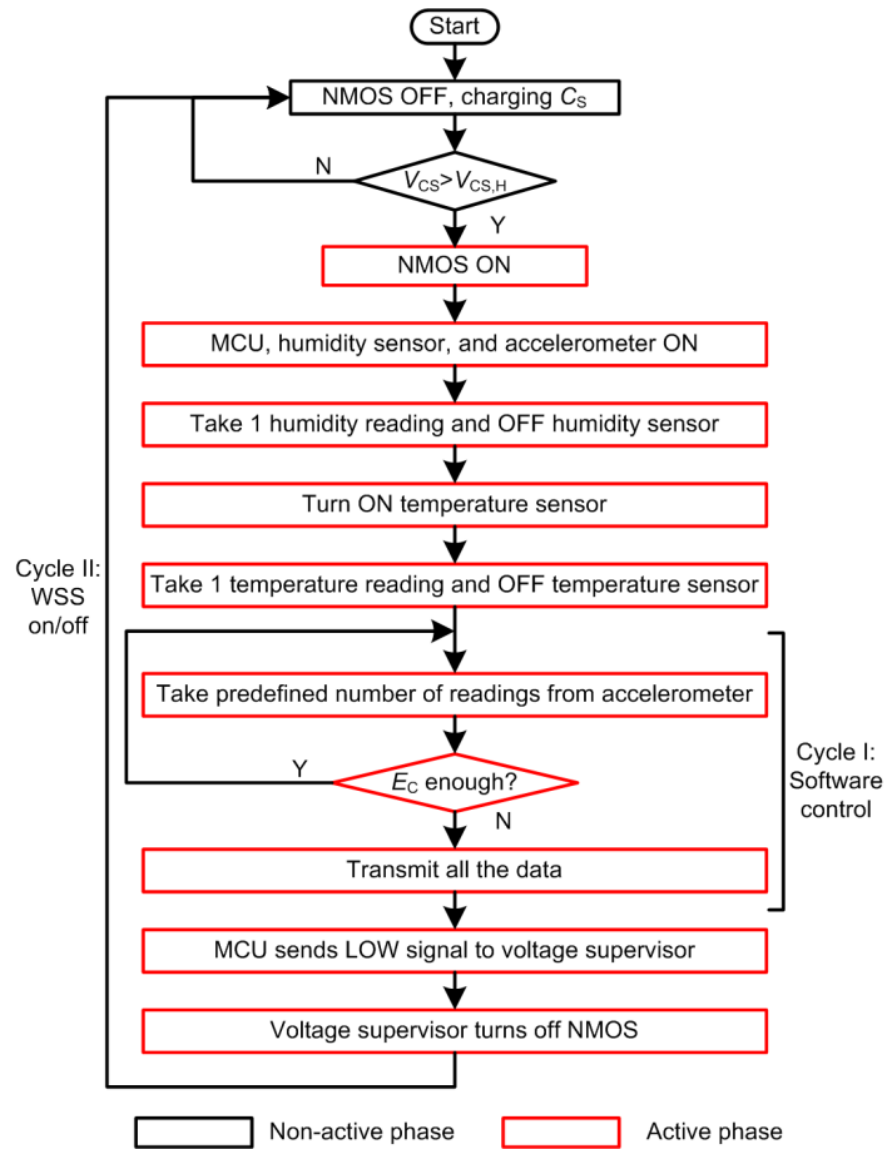

Fig. 4. Operation flowchart of the implemented WSS.

switch. The voltage supervisor has a selection of turn-on and turn-off threshold voltages but it can be instructed by the WSS to turn off the NMOS without having to wait for $V_{\mathrm{CS}}$ to reach the turn-off threshold voltage by using a software control which will be explained later in Section D [18].

Fig. 3 illustrates the operation of the EAI, where $V_{\mathrm{CS}, \mathrm{H}}$ and $V_{\mathrm{CS}, \mathrm{L}}$ are the turn-on and turn-off voltage thresholds of the voltage supervisor for turning-on and turning-off the WSS, respectively. It is assumed that (1), at the beginning, $C_{\mathrm{S}}$ is completely discharged with $V_{\mathrm{CS}}$ equals zero and the NMOS is switched off with the WSS in a non-active phase (denoted by $\mathrm{N}$ in Fig. 3), and (2) there is a continuous vibration across the SEH so that $C_{\mathrm{S}}$ can be charged up. The time duration required by $C_{\mathrm{S}}$ to be charged up from $0 \mathrm{~V}$ to the turn-on threshold $V_{\mathrm{CS}, \mathrm{H}}$ is called the cold start time, $t_{\mathrm{c}}$ here. When $V_{\mathrm{CS}}$ reaches $V_{\mathrm{CS}, \mathrm{H}}$, the NMOS is turned on by the voltage supervisor, connecting the negative terminal of the WSS to the system ground. $C_{\mathrm{S}}$ and WSS form a closed circuit which enables energy from $C_{\mathrm{S}}$ to flow into the WSS. The WSS therefore goes into an active phase (denoted by A in Fig. 3) to perform its sensing and data transmission tasks using the energy supplied from $C_{\mathrm{S}}$. $V_{\mathrm{CS}}$ will inevitably drop as energy is being consumed by the WSS. This period of time where the WSS becomes active is called the active time, $t_{\mathrm{a}}$ here. When $V_{\mathrm{CS}}$ drops to $V_{\mathrm{CS}, \mathrm{L}}$, the NMOS is turned off to disconnect the WSS from the system ground, effectively cutting off the energy supply. With no energy supply, the WSS goes into the non-active phase. $C_{\mathrm{S}}$ can then be charged up again by the SEH via the adaptive PMM. The system remains in the non-active phase until $V_{\mathrm{CS}}$ reaches $V_{\mathrm{CS}, \mathrm{H}}$ again and the aforementioned cycles repeat. The charging time from $V_{\mathrm{CS}, \mathrm{L}}$ to $V_{\mathrm{CS}, \mathrm{H}}$ is called the warm start time, $t_{\mathrm{w}}$ here.

The EAI controls $C_{\mathrm{S}}$ to provide a voltage between $V_{\mathrm{CS}, \mathrm{H}}$ and $V_{\mathrm{CS}, \mathrm{L}}$ to the WSS in an energy efficient manner because energy from $C_{\mathrm{S}}$ is directly transferred to the WSS without going through another system such as a DC-DC converter or linear regulator. The WSS has an operating voltage range of $2.4 \mathrm{~V}$ to $3.3 \mathrm{~V}$ in our studied case. Therefore, $V_{\mathrm{CS}, \mathrm{H}}$ of $3.16 \mathrm{~V}$ was chosen from the available threshold selections of the voltage supervisor and $V_{\mathrm{CS}, \mathrm{L}}$ will be determined by the software control to guarantee successful data transmission by the WSS before the energy becomes too low.

\section{Wireless Sensor System}

The WSS is composed of a wireless microcontroller (MCU) and three analogue sensors, as shown in Fig. 1(a) and (b). The MCU (Jennic JN5148) used is low power, compliant to IEEE 802.15.4 standard for communication using ZigBee protocol [4],[18] and has $128 \mathrm{kB}$ of random access memory (RAM). The sensors used are a humidity sensor (HIH-5030), a temperature sensor (MCP9700), and a 3-axis accelerometer (ADXL335). Therefore, the implemented WSS can make five measurements, which are the humidity, temperature, and accelerations from $x$-, $y$-, and $z$-axis. Outputs from all the sensors are directly connected to the analogue-to-digital converter (ADC) pins of the MCU for data reading.

Operation of the implemented WSS is illustrated using a flowchart, shown in Fig. 4. The operation begins with the WSS in the non-active phase until there is enough energy to turn on the WSS by the EAI as explained earlier. Once there is sufficient energy, the voltage supervisor turns on the NMOS, which in turn switches on the MCU, humidity sensor and accelerometer. The MCU takes one reading from the humidity sensor and turns it off before the temperature sensor is turned on for one temperature reading and then turned off for energy saving. After that, the MCU takes a total of three readings (one each from $x$-, $y$-, and $z$-axis) from the accelerometer every $10 \mathrm{~ms}$. The MCU repeatedly takes reading from the accelerometer until a pre-defined number of readings were made, for example, a total of 48 readings from 16 data sampling of each axis of the accelerometer to ensure there are enough readings to sample a vibration of low to medium frequency. Each reading is 2 bytes of data and is stored in the RAM immediately after each measurement. Then, the MCU measures $V_{\mathrm{CS}}$ to determine whether there is enough energy for the MCU to take another 48 readings from the accelerometer and transmit all the data stored in the RAM. If so, the MCU will take another 48 readings from the accelerometer and measures $V_{\mathrm{CS}}$ again. If there is not enough energy in the storage capacitor for the MCU to take another 48 readings, the MCU transmits all the data stored in the RAM and sends a LOW signal to the RST (reset pin) of the voltage supervisor.

The cycle I, as shown in Fig. 4, for taking another 48 reading is the software control where the MCU is programmed to judge whether there is sufficient energy for the WSS to take another 48 readings, which is essential to take as many reading 
as possible for dynamic measurements. The reason of taking 48 readings is because the maximum data size in one packet using ZigBee protocol is around 100 bytes [19]. The voltage supervisor then turns off the NMOS so that the WSS goes into the non-active phase and the cycle II, shown in Fig. 4, repeats, alternating between the active phase and non-active phase as long as there is energy from the SEH. The data size to be transmitted in the first packet is 100 bytes, which consists of the readings from all three sensors and the remaining packets only have 96 bytes from the 48 accelerometer readings.

It should be noted that only one-way communication from the sensor to the base station is considered here, due to the limited energy availability from energy harvesting. It is worthwhile to mention that it is common to use one-way communication in current energy harvesting powered systems [2], [3], [11] A retransmission mechanism which involves two-way communication between the node and the base station is not used to verify the success of the data transmission because it is usually very power hungry and there is need for further work on reducing that power consumption. The harvested energy should be mainly used for the sensing tasks instead of on the wireless communication. To reduce the failure rate in the wireless data transmission, the MCU was set to transmit the data using maximum power at $2.5 \mathrm{dBm}$.

\section{EXPERIMENTAL SETUP AND VERIFICATION METHODS}

\section{A. Experimental Setup}

The energy generation and distribution, system efficiency, and WSS powering capability of the developed system shown in Fig. 1(a), were evaluated experimentally. In order to evaluate the performance of the implemented system, comparative studies with other three different circuit configurations, as shown in Fig. 5 with their measuremental points, were performed. In CFG 1, the SEH was connected with a variable resistive load for characterization of the maximum power that can be potentially harvested from the SEH. In CFG 2, a smoothing capacitor was added in parallel with the resistive load in CFG 1, representing a more realistic and practical circuit design and was used to characterize the maximum power that can be potentially stored in a capacitor because a smoothing capacitor usually presents after the rectifier to regulate the rectified voltage in many circuit designs [15]-[17]. CFG 3 has a storage capacitor, an EAI, and a WSS, but without the adaptive PMM, which is similar to the system presented in [9], [11]. Both CFG 1 and 2 serve as references to determine the enhanced performances of the developed system in harvesting energy from the SEH. The adaptability of the developed system for maximum power harvesting was evaluated by comparing with CFG 3 to show the importance of using the adaptive PMM.

All the measurements were made using Keithley 2612B sourcemeter units which were connected to a computer. Data was transmitted wirelessly by the WSS to a base station which was connected to another computer positioned at about $4 \mathrm{~m}$ away to collect the wirelessly transmitted data. The circuits of CFGs 1 to 3 were built on breadboard for ease of implementation and measurements. The voltage and current generated by the SEH in CFGs 1 and 2 were measured in real time to determine the power while the variable resistor was being tuned until the maximum power was found.

An Instron E10000 ElectroPuls dynamic testing machine was used to apply different strain loadings at different frequencies onto the $\mathrm{SEH}$, which represents variable strain loading conditions on the aircraft wing.

\section{B. Energy Distribution and Efficiency}

The instantaneous power generated by the SEH is:

$$
p_{\mathrm{g}}(t)=v_{\mathrm{g}}(t) i_{\mathrm{g}}(t)
$$

The energy generated by the SEH and the average power were calculated as follows:

$$
\begin{gathered}
E_{\mathrm{g}}\left(t_{N}\right)=\sum_{k=1}^{N} v_{\mathrm{g}}\left(t_{k}\right) i_{\mathrm{g}}\left(t_{k}\right) \Delta t \\
P_{\mathrm{g}}=\frac{E_{\mathrm{g}}\left(t_{N}\right)}{t_{N}}
\end{gathered}
$$

where $\Delta t$ is the sampling period, which is $1 \mathrm{~ms}$ for all the cases in this paper. The energy and power generated from the SEH in all the circuit configurations were calculated using (2) to (3), but with the added suffices of 'r', 'rc', 'EAI' and 'PMM' representing CFG 1, CFG 2, CFG 3, and the developed system with adaptive PMM respectively. These suffice also show the types of circuits at the rectifier output.

Energy output from the rectifier $E_{\text {rect, }}$, the adaptive PMM $E_{\text {out-PMM, }}$ and energy consumed by the WSS $E_{\mathrm{W}}$ were calculated using the same equation as given in (2) by multiplying the voltage and current of the same subscript. Similarly, their respective average powers were obtained using (3). The efficiency of the rectifier $\eta_{\text {rect }}$ was calculated by (4).

$$
\eta_{\text {rect }}=\frac{P_{\text {rect }}}{P_{\mathrm{g}}} \times 100 \%
$$

The overall system efficiency $\eta_{\text {all }}$ of the system that takes energy from the SEH and transfer to the storage capacitor in CFG 1, 2, 3, and the developed system were calculated by (5):

$$
\eta_{\text {all }}=\frac{P_{\text {out }}}{P_{\mathrm{g}}} \times 100 \%
$$

It should be noted that $\eta_{\text {all }}$ and $\eta_{\text {rect }}$ for CFG 1 to 3 are the

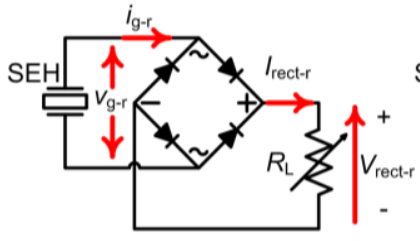

(a)

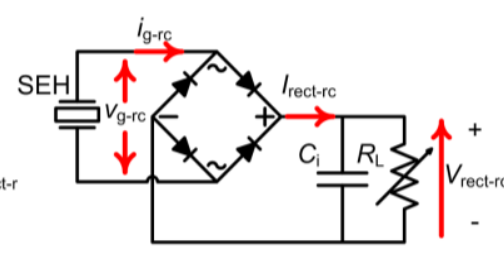

(b)

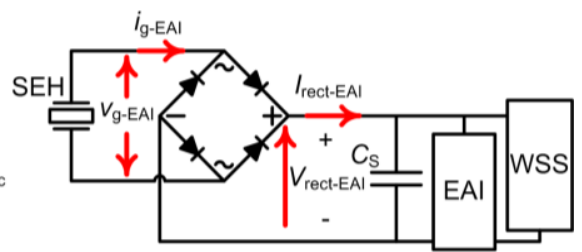

(c)

Fig. 5. Schematic of circuit configurations for (a) CFG 1, (b) CFG 2, and (c) CFG 3. 
same because the system interface which transfers the energy from the SEH to the storage capacitor in these circuit configurations is the rectifier itself. The capacitor current $I_{\mathrm{C}}$ PMM in the developed system was calculated by (6):

$$
i_{\text {C-PMM }}\left(t_{k}\right)=i_{\text {out-PMM }}\left(t_{k}\right)-i_{\mathrm{W}}\left(t_{k}\right)
$$

where $i_{\text {out-PMM }}$ and $i_{\mathrm{W}}$ are the currents from the output of the adaptive PMM and consumed by the EAI and WSS, respectively. The energy consumed by the WSS is mainly drawn out from the storage capacitor $C_{\mathrm{S}}$. Therefore, the energy remained in $C_{\mathrm{S}}$ of the developed system is given by (7).

$$
E_{\mathrm{C}-\mathrm{PMM}}\left(t_{k}\right)=E_{\text {out-PMM }}\left(t_{k}\right)-E_{\mathrm{W}}\left(t_{k}\right)
$$

The energy generation capability for all four circuit configurations was experimentally evaluated by applying cyclic loading onto the SEH using the Instron testing machine. The SEH was subjected to a peak-to-peak strain loading of $600 \mu \varepsilon$ at $10 \mathrm{~Hz}$. The smoothing capacitor $C_{\mathrm{i}}$ used in CFG 2 and the developed system is a $22 \mu \mathrm{F}$ capacitor. This value was chosen because according to the analysis of power transfer from the PEH to the load at the rectifier output, the size of the smoothing capacitor should be much larger than the intrinsic capacitance of the PEH for a higher energy harvesting efficiency [20] given that the SEH has an intrinsic capacitance of around $200 \mathrm{nF}$ [14]. The storage capacitor $C_{\mathrm{S}}$ used in the developed system and CFG 3 is a $10 \mathrm{mF}$ capacitor for the energy generation and energy distribution tests.

\section{Applied Strain Loading and Capacitor Size}

The purposes of the tests are to verify the energy harvesting and WSS powering capabilities of the developed system under different vibrational conditions. Therefore, only the developed system which is adaptive will be tested in this experiment. The test using different vibration conditions gives a thorough insight of the system performance. It also gives a realistic scenario which can evaluate the feasibility of the developed system to be deployed in real-world situations where the ambient vibration conditions can be highly variable.

For the strain tests, the SEH was subjected to peak-to-peak strain loadings of $300 \mu \varepsilon, 400 \mu \varepsilon, 500 \mu \varepsilon$, and $600 \mu \varepsilon$ at frequency of $10 \mathrm{~Hz}$. For the frequency tests, the SEH was subjected to a peak-to-peak strain loading of $600 \mu \varepsilon$ using different frequencies of 2, 4, 6, 8, and $10 \mathrm{~Hz}$. These conditions resemble the vibrations of the aircraft wing [7], [9].

All the aforementioned tests were conducted using a storage capacitor of $10 \mathrm{mF}$ first. Then, they were repeated using capacitor of $20 \mathrm{mF}$ and $30 \mathrm{mF}$ for understanding of the effects of capacitor size to the WSS powering capability. These values were chosen so that the WSS can be turned on for more than hundreds of milliseconds and up to a few seconds, which is normally required in real-world applications especially for measuring low frequency vibrations.

$P_{\text {g-PMM }}, P_{\text {rect-PMM }}, P_{\text {out-PMM }}, t_{\mathrm{c}}, t_{\mathrm{a}}, t_{\mathrm{w}}$, and data transmission of the WSS under all the different tested conditions were compared. The capability of SEH powering the WSS was accessed by the duty cycle of the WSS, which is defined as:

$$
D=\frac{t_{\mathrm{a}}}{t_{\mathrm{a}}+t_{\mathrm{w}}} \times 100 \%
$$

\section{RESUlts AND DISCUSSIONS}

\section{A. Energy Generated by the SEH}

Fig. 6 shows the energy generated by the SEH using all four different circuit configurations when they were subjected to a peak-to-peak strain loading of $600 \mu \varepsilon$ at $10 \mathrm{~Hz}$ in $60 \mathrm{~s}$. The optimal resistances found in CFGs 1 and 2 are $68.96 \mathrm{k} \Omega$ and $98 \mathrm{k} \Omega$ respectively. It can be seen that the SEH produced highest amount of energy at $301.9 \mathrm{~mJ}$ when it was connected to its optimal resistive load using CFG 1 . However, it should be noted that the energy produced in CFG 1 is dissipated by the resistor and not all the energy can be stored in the capacitor once the SEH is connected with a rectifier and a capacitor, which can be seen from the result of the SEH in CFG 2 that generates about $31.7 \%$ less energy than in CFG 1 at $206.2 \mathrm{~mJ}$. This is because there are times that current $i_{\mathrm{g}-\mathrm{rc}}$ from the SEH could not be delivered to the load at the rectifier output. The smoothing capacitor tends to hold the voltage $V_{\text {rect- }}$ rc constant at the rectifier output while the SEH voltage $v_{\mathrm{g}-\mathrm{rc}}$ is an AC voltage with varying amplitude. The diodes of the rectifier are reverse-biased and hence become non-conductive when the magnitude of $v_{\mathrm{g} \text {-rc }}$ is smaller than $V_{\text {rect-rc }}$, which stops the current flow from the SEH to the load at the rectifier output. Therefore, the energy that can be harvested from the

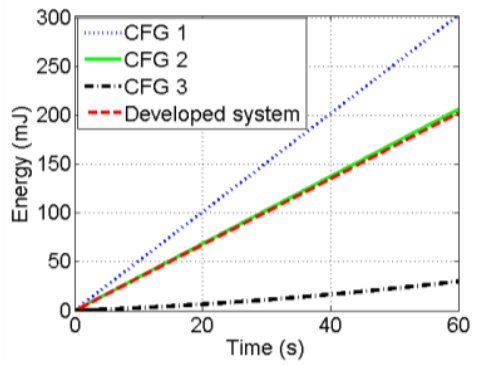

Fig. 6. Energy generated by the SEH using different circuit configurations when subjected to a peak-to-peak strain loading of $600 \mu \varepsilon$ at $10 \mathrm{~Hz}$ in $60 \mathrm{~s}$.

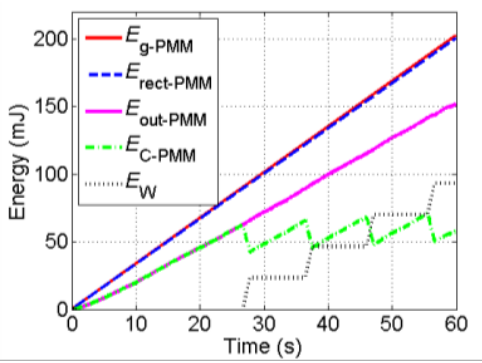

Fig. 7. Energy distribution of the developed system when the SEH was subjected to a peak-to-peak strain loading of $600 \mu \varepsilon$ at $10 \mathrm{~Hz}$ in $60 \mathrm{~s}$.

TABLE I

COMPARISONS OF THE SySTEMS WITH DiFFERENT CiRCUIT CONFIGURATIONS

\begin{tabular}{lccccc}
\hline \hline \multicolumn{1}{c}{ Configuration } & $\begin{array}{c}P_{\mathrm{g}} \\
(\mathrm{mW})\end{array}$ & $\begin{array}{c}P_{\text {rect }} \\
(\mathrm{mW})\end{array}$ & $\begin{array}{c}P_{\mathrm{C}} \\
(\mathrm{mW})\end{array}$ & $\begin{array}{c}\eta_{\text {rect }} \\
(\%)\end{array}$ & $\begin{array}{c}\eta_{\text {all }} \\
(\%)\end{array}$ \\
\hline CFG 1: $68.96 \mathrm{k} \Omega$ & 5.03 & 4.92 & - & 97.76 & 97.76 \\
$\mathrm{CFG} \mathrm{2:} C_{\mathrm{i}}+98 \mathrm{k} \Omega$ & 3.44 & 3.34 & - & 97.15 & 97.15 \\
CFG 3: $C_{\mathrm{S}}+\mathrm{EAI}+\mathrm{WSS}$ & 0.50 & 0.35 & 0.35 & 70.00 & 70.00 \\
$\begin{array}{l}\text { Developed system: } C_{\mathrm{i}}+\text { adaptive } \\
\text { PMM }+C_{\mathrm{S}}+\mathrm{EAI}+\mathrm{WSS}\end{array}$ & 3.38 & 3.34 & 2.53 & 99.00 & 75.00 \\
\hline \hline
\end{tabular}


SEH in CFG 2 is less than CFG 1. Similar to CFG 2, the developed system and CFG 3 have a capacitor at the rectifier output. So, the maximum power which can be harvested is no more than CFG 2. With the adaptive PMM introduced in the developed system, the harvested energy is very close to CFG 2 at $202.8 \mathrm{~mJ}$. Without the adaptive PMM as in CFG 3, the harvested energy is $30 \mathrm{~mJ}$. Despite the lower harvested energy, the WSS can be turned on in CFG 3 as in [9], [11] since energy can be accumulated in the storage capacitor until there is enough energy for the WSS operation. This shows the importance of using the adaptive and energy-aware interface as well as a full system integration study since high efficiency of a subsystem does not reflect the high energy harvesting capability of the whole system as can be seen in Table I. The developed system and CFG 3 have similar overall efficiencies but have significantly different amount of harvested power.

\section{B. Energy Distribution}

The developed system was further scrutinized to determine the energy distribution from the SEH to the WSS to understand the system efficiency. The voltage and current profiles of the WSS powered by the developed system were studied. Fig. 7 shows the energy distribution in the developed system when a strain loading of $600 \mu \varepsilon$ at $10 \mathrm{~Hz}$ was applied onto the SEH. The rectifier has a high efficiency of $99 \%$ and the adaptive PMM has an efficiency $\eta_{\mathrm{PMM}}$ of $75.6 \%$. After $26.68 \mathrm{~s}, 62.52 \mathrm{~mJ}$ of energy has been accumulated in $C_{\mathrm{S}}$, which is sufficient to turn on the WSS. During its active phase, the WSS consumes $23.29 \mathrm{~mJ}$ of energy, which sees $E_{\mathrm{W}}$ increases and the amount of energy in the storage capacitor $E_{\mathrm{C}}$ PMM reduces by $20.36 \mathrm{~mJ}$. The small difference between the energy consumed by the WSS and energy reduction in $C_{\mathrm{S}}$ is due to a small amount of energy consumed by the WSS is provided directly by the adaptive PMM. $E_{\mathrm{C}-\mathrm{PMM}}$ does not reduce to zero since there is some energy remained in $C_{\mathrm{S}}$ as the WSS is turned off at $2.47 \mathrm{~V}$ in this case, as determined by the software control. After the active phase, the WSS goes into the non-active phase. $E_{\mathrm{W}}$ remains the same as there is no energy consumed by the WSS in this phase.

The voltages and currents of the system at different stages are shown in Fig. 8, which can be used to explain the trends observed in the energy distribution shown in Fig. 7. Fig. 8(a) shows the measured $v_{\mathrm{g}-\mathrm{PMM}}$ and $V_{\text {rect-PMM }}$ in the developed system where the peak magnitude difference between $v_{\text {g-PMM }}$ and $V_{\text {rect-PMM }}$ due to the forward voltage drop $V_{\mathrm{F}}$ across the rectifier is less than $1.0 \mathrm{~V}$, as shown in the inset of Fig. 8(a).

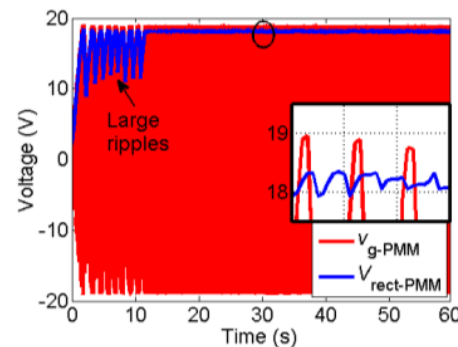

(a)

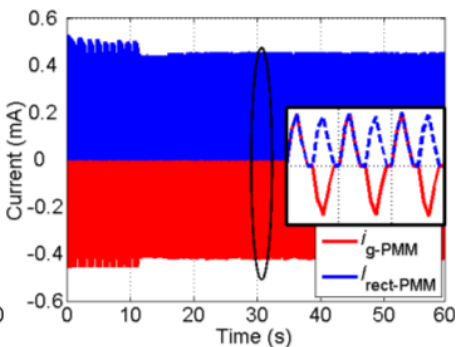

(b)
$V_{\mathrm{F}}$ is only a small fraction of $v_{\mathrm{g}-\mathrm{PMM}}$ which has a peak amplitude of $19 \mathrm{~V}$. Current $I_{\text {rect-PMM }}$ is the rectified $i_{\mathrm{g} \text {-PMM }}$, as shown in Fig. 8(b), where its inset shows that $I_{\text {rect-PMM }}$ overlaps $i_{\mathrm{g} \text {-PMM }}$ at the positive polarity, indicating no reduction in current passing through the rectifier. Therefore, the energy loss in the rectifier is very small because of the relatively small voltage loss across it compared with the high voltage and low current going through it. It should be noted that the large ripples in Fig. 8(a) and (b) during the initial time of $10 \mathrm{~s}$ is due to the Instron machine requires some time to provide a steady output, which is not caused by the developed system.

The high voltage of $v_{\mathrm{g}-\mathrm{PMM}}$ and low current of $i_{\mathrm{g}-\mathrm{PMM}}$ generated by the SEH is converted by the adaptive PMM into an output with lower voltage $V_{\text {out-PMM }}$ and higher current $i_{\text {C-PMM }}$ to the storage capacitor, as shown in Fig. 8(c) and (d). The WSS becomes active when $V_{\text {out-PMM }}$ reaches $3.16 \mathrm{~V}$, which sees a sudden increase in the voltage $V_{\mathrm{W}}$ across the WSS and current $I_{\mathrm{W}}$ drawn by the WSS. More current is required for data transmisson at the end of $t_{\mathrm{a}}$. Therefore, the WSS can be regarded as a variable electrical load and the developed system is adaptive to it. The WSS stays active until $V_{\text {out-PMM }}$ drops to $2.47 \mathrm{~V}$ and goes back to its non-active phase. $I_{\mathrm{W}}$ becomes approximately zero while $V_{\mathrm{W}}$ drops to approximately $0.6 \mathrm{~V}$ and gradually decreases during the warm start time $t_{\mathrm{w}}$ because there is a small decoupling capacitor across the WSS which tends to hold the voltage. $i_{\mathrm{C}-\mathrm{PMM}}$ is a good representative of $I_{\text {out-PMM }}$ based on (6) because $I_{\mathrm{W}}$ is around $0.95 \mu \mathrm{A}$ for most of the time during $t_{\mathrm{c}}$ and $t_{\mathrm{w}}$, as shown in the inset of Fig. 8(d). This shows the EAI consumes very low current to keep the WSS off during the non-active phase until the energy in $C_{\mathrm{S}}$ is sufficient to meet the energy demanded by the WSS. During the active phase, $i_{\mathrm{C}-\mathrm{PMM}}$ becomes negative for most of the time because most of the current required by the WSS to stay active is flowing out from $C_{\mathrm{S}}$. Therefore, $I_{\mathrm{W}}$ becomes positive with a similar peak magnitude at the same time.

\section{Effects of Different Applied Mechanical Loadings}

Fig. 9 shows the measuremental results of (a) $P_{\text {g-PMM }}$, (b) $P_{\text {rect-PMM }}$, (c) $P_{\text {out-PMM }}$, (d) duty cycle, (e) $t_{\mathrm{c}}$, (f) $t_{\mathrm{w}},(\mathrm{g}) t_{\mathrm{a}}$, and (h) data transmitted in the developed system using different strain levels, frequencies, and capacitor sizes. It can be seen from Fig. 9(a) to (c) that the power harvested mainly varies with the mechanical loading applied onto the SEH and does not vary much with the connected capacitor of different sizes. This means that the power harvesting capability of the developed system is insensitive to the connected electronics at its output,

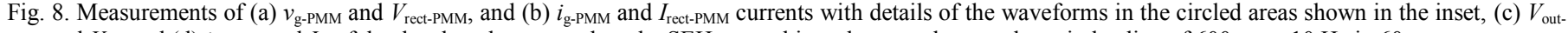
PMM and $V_{\mathrm{W}}$, and (d) $i_{\mathrm{C}-\mathrm{PMM}}$ and $I_{\mathrm{W}}$ of the developed system when the $\mathrm{SEH}$ was subjected to a peak-to-peak strain loading of $600 \mu \varepsilon$ at $10 \mathrm{~Hz}$ in $60 \mathrm{~s}$. 
which include the capacitor and WSS. Therefore, it can be said that the introduced adaptive PMM and EAI makes the developed system adaptive towards the variations of the mechanical loadings applied to the SEH and the variation of the connected end devices. In addition, it can also be seen that higher strain loadings and higher frequencies applied onto the SEH resulted in higher generated power and vice versa.

The power output by the adaptive PMM is relatively proportional to the input power $P_{\text {rect-PMM }}$ to the adaptive PMM without much influenced by different capacitor sizes. The duty cycle calculated based on $t_{\mathrm{a}}$ and $t_{\mathrm{w}}$ is directly related to the power generated by the SEH as can be seen from Fig. 9(d). The duty cycle follows the trends in Fig. 9(a) where it is highest when $P_{\text {g-PMM }}$ is highest and vice versa, which is common in energy harvesting powered systems to ensure long term operation of the WSS based on the energy level [4].

\section{Effects of Different Capacitor Sizes}

Fig. 9 shows that the capacitor size has negligible effect on $P_{\text {g-PMM }}, P_{\text {rect-PMm }}, P_{\text {out-PMm, }}$ and the duty cycle since they are about the same for a specific test condition regardless of the capacitor size. The capacitor size mainly affects the cold start time $t_{\mathrm{c}}$, warm start time $t_{\mathrm{w}}$, and active time $t_{\mathrm{a}}$. The time required to charge up different sizes of capacitor varies with $P_{\text {out-PMM }}$ which changes with the strain level and frequency experienced by the SEH. The increment in $t_{\mathrm{c}}$ and $t_{\mathrm{w}}$ are rather proportional to the increment of capacitor size, as shown in Fig. 9(e) and (f), respectively. $t_{\mathrm{a}}$ also has a similar trend, which increases by a factor of increment in capacitor size but is not much influenced by $P_{\text {out-PMM }}$ because $t_{\mathrm{a}}$, which is also the discharging time of the capacitor is mainly determined by the power consumption of the WSS. Therefore, a larger capacitor is able to keep the WSS active for a longer period of time and vice versa. The active time of about 1 second or longer allows vibration frequency of lower than $1 \mathrm{~Hz}$ to be sampled.

There are slight variations in $t_{\mathrm{a}}$ with different test conditions, where $t_{\mathrm{a}}$ slightly increases with higher strain levels

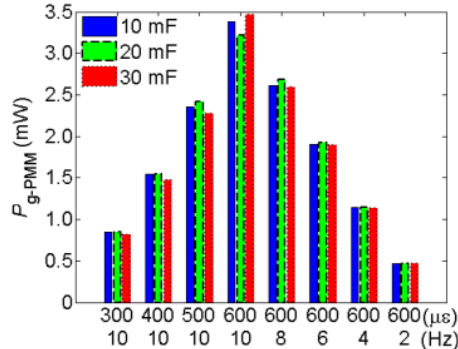

(a)

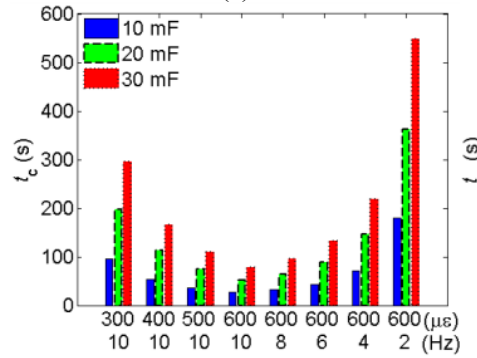

(e)

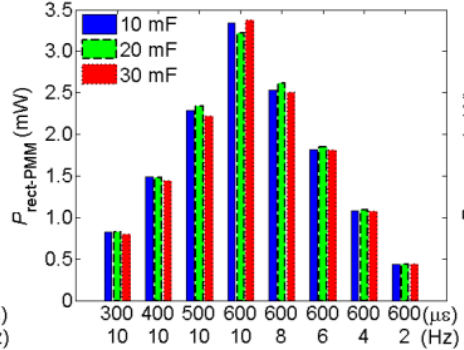

(b)

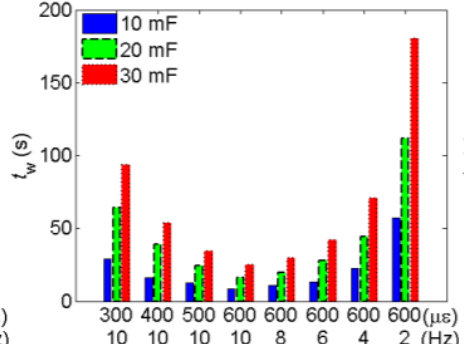

(f) or frequencies. This is because the adaptive PMM is still transferring energy from the SEH to the storage capacitor and then to WSS during $t_{\mathrm{a}}$ as discussed earlier and shown in Fig. 8(d). The SEH produces higher power when higher strain loadings and frequencies are applied onto it, as shown in Fig. 9(a). Therefore, more energy is available to the WSS especially during high strain and frequency test conditions which slightly prolongs its active time. Although the duty cycle is determined by $t_{\mathrm{a}}$ and $t_{\mathrm{w}}$, it remains approximately the same without much influenced by the capacitor size because $t_{\mathrm{a}}$ and $t_{\mathrm{w}}$ change approximately by the same factor with the change in the capacitor size for a given input power.

The data size that can be transmitted by the WSS is directly related to $t_{\mathrm{a}}$. Therefore, Fig. 9(h) exhibits a similar trend as in Fig. $9(\mathrm{~g})$. The total data size for the five measurements are 484 bytes, 1060 bytes, and 1540 bytes for using capacitor size of $10 \mathrm{mF}, 20 \mathrm{mF}$, and $30 \mathrm{mF}$, respectively, which are quite proportional to the increment in the capacitor size. If only one directional vibration is measured, with the data size that can be measured and transmitted as discussed earlier, the developed system can be used to measure vibration of up to $120 \mathrm{~Hz}$ for structural health monitoring or condition monitoring [5].

In general, although the size of capacitor has an impact on $t_{\mathrm{c}}, t_{\mathrm{a}}$, and $t_{\mathrm{w}}$, it has a negligible effect on the duty cycle. It mainly affects the operational time of the WSS by defining the operation duration $\left(t_{\mathrm{a}}\right)$ and how frequent is the operation within a time frame $\left(1 /\left(t_{\mathrm{a}}+t_{\mathrm{w}}\right)\right)$. For example, reducing the size can shorten the charging time of $t_{\mathrm{c}}$ and $t_{\mathrm{w}}$ which allows the WSS to operate more frequently in a given time frame but this resulted in a reduced $t_{\mathrm{a}}$, and subsequently reduced amount of measured data. If the harvested energy is high, the charging time of $t_{\mathrm{c}}$ and $t_{\mathrm{w}}$ can be reduced while $t_{\mathrm{a}}$ of the WSS can be increased. Therefore, it is important to harvest the maximum possible energy from the energy harvester and transfer the energy to the storage capacitor with high efficiency.

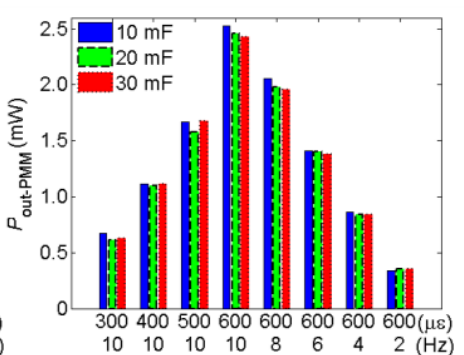

(c)

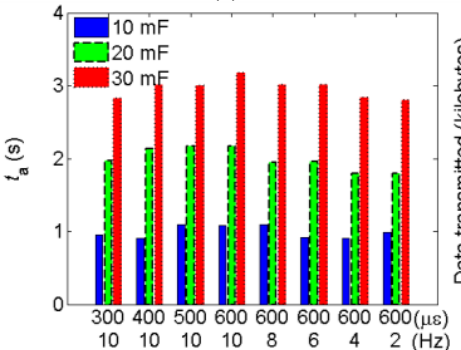

(g)

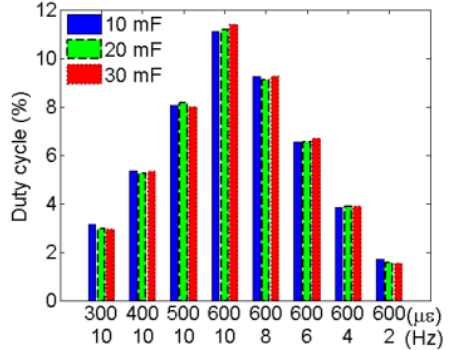

(d)

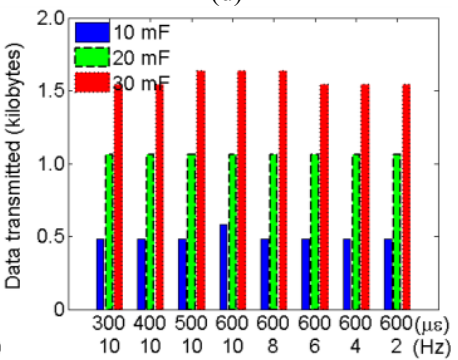

(h)

Fig. 9. Measurements of (a) $P_{\text {g-PMm, }}$ (b) $P_{\text {rect-PMm, }}$, (c) $P_{\text {out-PMm, }}$, (d) duty cycle, (e) $t_{\mathrm{c}}$, (f) $t_{\mathrm{w}},(\mathrm{g}) t_{\mathrm{a}}$, and (h) data transmitted in the developed system using different peak-to-peak strain levels (numbers on the top of x-axis), frequencies (numbers on the bottom of $\mathrm{x}$-axis), and capacitor sizes. 
TABLE II

Summary of The PERformances of THE DeVEloped System With DifFERENT CAPACITOR Sizes

\begin{tabular}{|c|c|c|c|c|c|c|c|c|c|}
\hline $\begin{array}{l}\text { Capacitor } \\
\text { size }(\mathrm{mF})\end{array}$ & $\begin{array}{c}P_{\mathrm{g}-\mathrm{PMM}} \\
(\mathrm{mW})\end{array}$ & $\begin{array}{l}P_{\text {rect-PMM }} \\
(\mathrm{mW})\end{array}$ & $\begin{array}{c}P_{\text {out-PMM }} \\
(\mathrm{mW})\end{array}$ & $\begin{array}{l}\eta_{\text {all }} \\
(\%)\end{array}$ & $\begin{array}{l}t_{\mathrm{c}} \\
(\mathrm{s})\end{array}$ & $\begin{array}{l}t_{\mathrm{w}} \\
(\mathrm{s})\end{array}$ & $\begin{array}{l}t_{\mathrm{a}} \\
(\mathrm{s})\end{array}$ & $\begin{array}{c}\text { Duty cycle } \\
(\%)\end{array}$ & $\begin{array}{c}\text { Data transmitted } \\
(\mathrm{kB})\end{array}$ \\
\hline 10 & $0.47-3.38$ & $0.44-3.34$ & $0.34-2.53$ & $70.76-78.93$ & $26.68-179.56$ & $8.65-57.41$ & $0.91-1.09$ & $1.70-11.10$ & $484-580$ \\
\hline 20 & $0.47-3.29$ & $0.44-3.22$ & $0.35-2.46$ & $70.42-76.40$ & $51.81-361.68$ & $16.14-111.71$ & $1.79-2.17$ & $1.58-11.85$ & $1060-1156$ \\
\hline 30 & $0.47-3.46$ & $0.44-3.37$ & $0.35-2.43$ & $70.23-76.83$ & $77.43-548.20$ & $24.72-180.50$ & $2.80-3.18$ & $1.53-11.40$ & $1540-1636$ \\
\hline
\end{tabular}

\section{E. System Efficiency and WSS Powering Capability}

It is worthwhile to highlight that the overall system efficiency of the developed system using the different strain loadings, vibration frequencies, and capacitor sizes is between 70 and $80 \%$ for all the tested conditions, calculated using (5) based on the results from Fig. 9(a) and (c). The duty cycles calculated in all the tested conditions are more than $1 \%$ where the highest one is more than $11 \%$. The large storage capacitors can be charged up within a realistic amount of waiting time of less than 10 minutes for all the tested conditions, which suits the needs of structural health monitoring which is done periodically [12]. The performances of the developed system are summarized in Table II.

It can be deduced that all the obtained performances of the implemented system are mainly attributed to the introduced blocks of adaptive PMM and the EAI. The adaptive PMM enables the power harvested to be close to the maximum possible amount of power that can be generated by the SEH which is interfaced with a rectifier and a smoothing capacitor in variable vibration conditions. The EAI offers an efficient energy flow management which ensures that there is no wastage of energy by keeping the WSS off while the energy is being accumulated in the storage capacitor until it is sufficient to meet the energy demand of the WSS.

Also, it can be seen that for a given amount of harvested power, the operation duration and operation frequency of the WSS as defined by $t_{\mathrm{a}}$ and $t_{\mathrm{w}}$ are related to the size of capacitor. Therefore, it is necessary to determine an optimal range of capacitance based on the intended task.

The minimum capacitor size depends on the minimum frequency $f_{\text {vib }}$ of the vibration to be measured. The capacitor has to provide sufficient energy for the WSS to stay active and sample at least one complete cycle of the vibration. Therefore, the minimum active time $t_{\mathrm{a}, \min }$ is given by (9):

$$
t_{\mathrm{a}, \min }=\frac{1}{f_{\mathrm{vib}}}
$$

It should be noted that the energy required $E_{\text {required }}$ by the WSS to stay active has to be available within the turn-on and turn off threshold voltages set by the EAI, as shown in (10):

$$
\frac{1}{2} C_{\mathrm{S}} V_{\mathrm{CS}, \mathrm{H}}^{2}-\frac{1}{2} C_{\mathrm{S}} V_{\mathrm{CS}, \mathrm{L}}^{2} \geq E_{\text {required }}=P_{\mathrm{W}} t_{\mathrm{a}}
$$

The power consumed $P_{\mathrm{W}}$ by the WSS for a particular task can be determined experimentally by setting the WSS to perform the task and measuring its power consumption [18]. The power consumption can be assumed to be constant for a specific implemented system. With $P_{\mathrm{W}}$ and $t_{\mathrm{a}, \min }$ known, substituting these values into (10) and rearranging (10) yields
(11), which is the minimum capacitor size $C_{\mathrm{S}, \min }$ required.

$$
C_{\mathrm{S}, \text { min }}=\frac{P_{\mathrm{W}} t_{\mathrm{a}, \min }}{\frac{1}{2} V_{\mathrm{CS}, \mathrm{H}}^{2}-\frac{1}{2} V_{\mathrm{CS}, \mathrm{L}}^{2}}
$$

The maximum capacitor size depends on the minimum operation frequency of the WSS. This operation frequency is mainly determined by the maximum allowable time duration of the WSS in non-active phase before becoming active again. This time is also the maximum allowable warm start time $t_{\mathrm{w}, \max }$, which increases with the capacitor size as discussed earlier in Section IV.D. Assume that the power output from the adaptive PMM $P_{\text {out-PMM }}$ to charge up $C_{\mathrm{S}}$ is known, (11) can be rewritten as (12) to determine the maximum capacitor size:

$$
C_{\mathrm{S}, \max }=\frac{P_{\mathrm{Out}-\mathrm{PMM}} t_{\mathrm{w}, \max }}{\frac{1}{2} V_{\mathrm{CS}, \mathrm{H}}^{2}-\frac{1}{2} V_{\mathrm{CS}, \mathrm{L}}^{2}}
$$

\section{CONCLUSION}

A fully integrated system of SEH powered WSS by using an adaptive and energy-aware interface for enhanced performance is presented in this paper. Introduction of the adaptive and energy-aware interface in the developed system increases the energy harvested from the SEH by around $670 \%$ from $0.5 \mathrm{~mW}$ to $3.38 \mathrm{~mW}$, when a peak-to-peak strain loading of $600 \mu \varepsilon$ at $10 \mathrm{~Hz}$ was applied onto the SEH. Such improvement allows the system to charge up a large storage capacitor of more than $10 \mathrm{mF}$ more effectively than a circuit without the adaptive PMM. The large storage capacitor allows the WSS to have a long active time and subsequently able to make dynamic measurements of low frequencies. Different storage capacitor sizes affect the cold start time, warm start time, active time, and the number of data bytes that can be transmitted. Therefore, the size of storage capacitor has to be chosen properly based on the intended applications. Energy can be transferred at high efficiency of 70-80 \% under various test conditions from the SEH to the output of the adaptive PMM for storage or used by the WSS. Also, with the efficient energy flow management achieved using the EAI and increased amount of harvested energy using the adaptive PMM, the WSS was successfully turned on to perform the programmed task under all the tested conditions with a duty cycle as high as $11.85 \%$. The importance of a whole system integration study is clearly seen in this paper because performances can be improved as demonstrated in energy generation, system efficiency, and WSS powering capability of the developed system for practical deployment of SEH powered WSNs in real-world scenarios. 


\section{REFERENCES}

[1] G. Park, T. Rosing, M. D. Todd, C. R. Farrar, and W. Hodgkiss, "Energy harvesting for structural health monitoring sensor networks," $J$. Infrastruct. Syst., vol. 14, no. 1, pp. 64-79, 2008.

[2] S. Sudevalayam, and P. Kulkarni, "Energy harvesting sensor nodes: Survey and implications," IEEE Commun. Surv. Tut., vol. 13, no. 3, pp. 443-461, 2011.

[3] A. E. Abdulhadi, and R. Abhari, "Multiport UHF RFID-tag antenna for enhanced energy harvesting of self-powered wireless sensors," IEEE Trans. Ind. Informat., vol. 12, no. 2, pp. 801-808, 2016.

[4] M. Magno, D. Boyle, D. Brunelli, E. Popovici, and L. Benini, "Ensuring survivability of resource-intensive sensor networks through ultra-low power overlays," IEEE Trans. Ind. Informat., vol. 10, no. 2, pp. 946-956, 2014.

[5] A. Nechibvute, A. Chawanda, and P. Luhanga, "Piezoelectric energy harvesting devices: An alternative energy source for wireless sensors," Smart Mater. Res., vol. 2012, pp. 853481 (13 pp), 2012.

[6] A. Emad, M. A. E. Mahmoud, M. Ghoneima, and M. Dessouky, "Testing and evaluation of stretching strain in clamped-clamped beams for energy harvesting," Smart Mater. Struct., vol. 25, no. 11, pp. 115006 (9 pp), 2016.

[7] M. Pozzi, S. Guo, and M. Zhu, "Harvesting energy from the dynamic deformation of an aircraft wing under gust loading," in Proc. SPIE Health Monit. Struct. Biol. Syst., 2012, vol. 8348, pp. 834831-1-11.

[8] D. A. van den Ende, H. J. van de Wiel, W. A. Groen, and S. van der Zwaag, "Direct strain energy harvesting in automobile tires using piezoelectric PZT-polymer composites," Smart Mater. Struct., vol. 21, no. 1, pp. 015011 (11 pp), 2012.

[9] A. Giuliano, V. Marsic, and M. Zhu, "Implementation and testing of an elastic strain powered wireless sensing system for energy-autonomous applications," in IEEE Int. Conf. Green Comput. Commun. (GreenCom), 2012, pp. 681-684.

[10] Y. Yang, L. Tang, and H. Li, "Vibration energy harvesting using macrofiber composites," Smart Mater. Struct., vol. 18, no. 11, pp. 115025 (8 pp), 2009.

[11] D. L. Churchill, M. J. Hamel, C. P. Townsend, and S. W. Arms, "Strain energy harvesting for wireless sensor networks," in Proc. SPIE Smart Struct. Mater., 2003, vol. 5055, pp. 319-327.

[12] N. De Battista, J. A. Rice, S.-H. Sim, J. M. W. Brownjohn, and P. H. Tan, "Embedded data processing in wireless sensor networks for structural health monitoring," Structural health monitoring 2013: A roadmap to intelligent structures, F.-K. Chang, Ed. DEStech Publications, 2013, pp. 1567-1574.

[13] S. Jang, H. Jo, S. Cho, K. Mechitov, J. A. Rice, S.-H. Sim, H.-J. Jung, C.-B. Yun, B. F. Spencer, Jr., and G. Agha, "Structural health monitoring of a cable-stayed bridge using smart sensor technology: Deployment and evaluation," Smart. Struct. Syst., vol. 6, no. 5-6, pp. 439-459, 2010.

[14] M. Pozzi, A. Canziani, I. Durazo-Cardenas, and M. Zhu, "Experimental characterisation of macro fibre composites and monolithic piezoelectric transducers for strain energy harvesting," in Proc. SPIE Health Monit. Struct. Biol. Syst., 2012, vol. 8348, pp. 834832-1-10.

[15] A. Tabesh, and L. G. Fréchette, "A low-power stand-alone adaptive circuit for harvesting energy from a piezoelectric micropower generator," IEEE Trans. Ind. Electron., vol. 57, no. 3, pp. 840-849, 2010.

[16] M. Shim, J. Kim, J. Jeong, S. Park, and C. Kim, "Self-powered $30 \mu \mathrm{W}$ to $10 \mathrm{~mW}$ piezoelectric energy harvesting system with $9.09 \mathrm{~ms} / \mathrm{V}$ maximum power point tracking time," IEEE J. Solid-State Circuits, vol. 50, no. 10, pp. 2367-2379, 2015.

[17] Z. J. Chew, and M. Zhu, "Microwatt power consumption maximum power point tracking circuit using an analogue differentiator for piezoelectric energy harvesting," JPCS, vol. 660, no. 1, pp. 012022 (5 pp), 2015.

[18] T. Ruan, Z. J. Chew, and M. Zhu, "Energy-aware approaches for energy harvesting powered wireless sensor nodes," IEEE Sensors J., vol. 17, no. 7, pp. 2165-2173, 2017.

[19] G. Anastasi, M. Conti, and M. D. Francesco, “A comprehensive analysis of the MAC unreliability problem in IEEE 802.15.4 wireless sensor networks," IEEE Trans. Ind. Informat., vol. 7, no. 1, pp. 52-65, 2011.

[20] W. J. Wu, A. M. Wickenheiser, T. Reissman, and E. Garcia, "Modeling and experimental verification of synchronized discharging techniques for boosting power harvesting from piezoelectric transducers," Smart Mater. Struct., vol. 18, no. 5, pp. 055012 (14 pp), 2009.

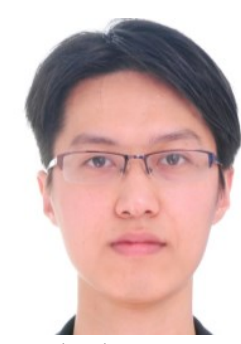

Zheng Jun Chew received his BEng degree in 2010 from the University of Strathclyde, Glasgow and $\mathrm{PhD}$ degree in 2014 at Swansea University, UK. He joined the Energy Harvesting Research Group at the University of Exeter in the UK, in 2014 as an Associate Research Fellow. Prior to pursuing his $\mathrm{PhD}$, he worked as an electrical engineer in Sony EMCS (M) Sdn. Bhd., Malaysia (2010).

His current research interest is in the area of power management module for energy-harvesting devices using novel and low power techniques.

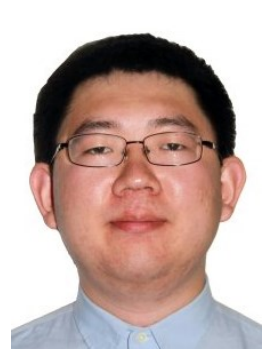

Tingwen Ruan received his BEng degree in 2012 from the Nanjing University of Posts and Telecommunications, China. He joined the Energy Harvesting Research Group at the University of Exeter in the $\mathrm{UK}$, in 2014 as a $\mathrm{PhD}$ student. Prior to starting his $\mathrm{PhD}$ study, he worked as a technical support engineer in the Zhongxing Telecommunication

Equipment Corporation, China (2013).

His current research interest is in the area of energy harvesting powered wireless sensing nodes and networks for structural health and environmental monitoring.

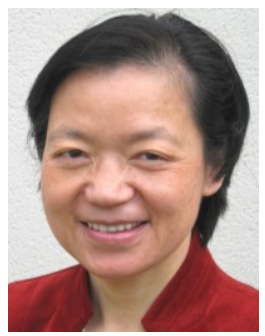

Meiling Zhu received her BEng degree in 1989, MEng in 1992, and PhD in 1995 at Southeast University, Nanjing, China. She currently holds the Professor and the Chair in Mechanical Engineering and the Head of Energy Harvesting Research Group in the University of Exeter in the UK. Prior to joining the University of Exeter, She worked in a number of Universities: Cranfield University (2002-13), the University of Leeds (2001-2); Stuttgart Universität (1999-2001); the Hong Kong University of Science and Technology (1998-9); and the Institute of Vibration Engineering Research, in the Nanjing University of Aeronautics \& Astronautics (1994-98).

Her current research interests are in the area of energy harvesting powered wireless sensor nodes for applications. 